\title{
Recursos psicosociales y construcción de bienestar ante la ausencia de los padres*
}

Psychological Resources and Construction of Welfare before the Absence of Parents

Recepción: 29 Mayo 2015 | Aprobación: 01 Diciembre 2017

\author{
Nancy Castaño Hincapié ${ }^{a}$ \\ Universidad Católica Luis Amigó, Colombia \\ ORCID: http://orcid.org/0000-0002-9241-6243 \\ Diana Marcela Montoya Zuluaga \\ Universidad Católica de Oriente, Colombia \\ ORCID: http://orcid.org/0000-0002-7674-1222 \\ Norman Darío Moreno Carmona \\ Universidad Católica Luis Amigó, Colombia \\ ORCID: http://orcid.org/0000-0002-8216-2569
}

\footnotetext{
a Autor de correspondencia. Correo electrónico: nancycastahincapie@gmail.com

Para citar este artículo: Castaño Hincapié, N., Montoya Zuluaga, D. M., \& Moreno Carmona, N. D. (2018). Recursos psicosociales y construcción de bienestar ante la ausencia de los padres. Universitas Psychologica, 17(2), 1-10. $\quad$ https://doi.org/10.11144/

Javeriana.upsy.17-2.rpcb
}

\section{RESUMEN}

El objetivo de este trabajo fue indagar sobre los recursos psicosociales asociados al bienestar subjetivo de adultos que experimentaron sentimientos de ausencia de alguno de los padres. Se aplicó entrevista semiestructurada y algunas láminas del Test de Apercepción Temática (TAT) a nueve personas que voluntariamente aceptaron participar y cumplían con los criterios de inclusión. Se encontró que los recursos psicosociales mediaron para que las personas, a pesar de haber experimentado ausencia de alguno de los padres, construyeran bienestar subjetivo. Esto es de suma significación, pues, en la medida en que se ofrezca a niños y jóvenes otros medios o recursos para hacerle frente a los sentimientos de ausencia, habrá más posibilidades de construir bienestar ante una realidad de padres cada vez más ausentes.

\section{Palabras clave}

bienestar subjetivo; familia; recursos psicosociales; sentimientos de ausencia.

\footnotetext{
ABSTRACT

The main objective of this survey was to inquire about the psychosocial resources, related with the subjective wellbeing of adults who experienced absence of some of the parents. We carried out semi-structured interview and some Thematic Apperception Test (TAT) prints to 9 nine people that freely accepted to participate and fulfilled the inclusion criteria. We found that the psychosocial resources were means so that the people, even if they experienced absence of some of their parents, could build a subjective wellbeing. This is quite relevant, because as far as we can offer to kids and young people some other possibilities or resources to face the feelings of absence, there will be more possibilities to build up wellbeing in front of Parents increasingly absent.

Keywords

subjective wellbeing; family; psychosocial resources; feelings of absence.
} 
Ante las múltiples modalidades de familia que hoy conocemos, los roles y funciones de los padres han venido sufriendo transformaciones, y el modelo de familia nuclear es cada vez más escaso y difícil de sostener. Profamilia (2010) muestra cifras en las cuales la proporción de niños que vive con ambos padres está disminuyendo. En la Encuesta Nacional de Demografía y Salud, ENDS (Profamilia, 2015), se menciona que en Colombia sigue el incremento de los hogares monoparentales, especialmente los de jefatura femenina. Un $12.6 \%$ de los hogares del país están conformados por familias nucleares monoparentales (falta el padre o la madre), y en el 2010, esta cifra era del $12.3 \%$. Lo anterior no significa que no vaya a ser posible mantener los vínculos familiares, o que los hijos vayan a padecer algún tipo de síntomas o desestabilizaciones, sino que se trata de investigar con cuáles otros recursos, alternativos a las familias, cuentan los sujetos para lograr bienestar subjetivo. Según las cifras anteriores, en la actualidad hay cada vez más familias donde los hijos no están lo suficientemente acompañados por sus padres; sin embargo, según el relato de las personas entrevistadas para la investigación, han logrado construir un proyecto de vida satisfactorio que les ha permitido asumir diferentes roles (padres, profesionales, estudiantes, trabajadores) que los llena de bienestar.

En un reporte sobre la familia, la revista Semana (2016) recoge los relatos de una pareja de antropólogos que investigaron la familia en diferentes culturas, y encontraron que los padres no ejercen tanta influencia en el desarrollo de los hijos como se cree. Según el país, los padres le dan importancia a diferentes formas de crianza o acompañamiento.

Coontz (1987) y Cebotarev (2001) proponen la familia como un proceso dinámico que posibilita que los procesos humanos se desarrollen normalmente (citados por Cebotarev, 2003). The Family Watch (2015) menciona los efectos negativos que puede representar la ausencia del padre en la salud y bienestar de los niños como diferentes formas de violencia y comportamiento asocial; también lo asocian con el consumo de drogas y la inestabilidad emocional. Sin embargo, en la realidad se encuentran personas que, a pesar de haber tenido problemas en las relaciones con los padres, al parecer, hubo algo más fuerte que les ayudó a contrarrestar estos sentimientos y salir victoriosos, logrando bienestar. García-Alandete (2016) señala que "hay personas que superan situaciones severamente adversas y que, incluso más allá, logran transformarlas en oportunidades para el crecimiento personal" (p. 21)

El bienestar subjetivo ha sido investigado recientemente desde la psicología positiva, en la que se evalúan aspectos como autonomía, satisfacción con la vida y optimismo (Pérez et al., 2011). Por ejemplo, Gómez, Villegas, Barrera y Cruz (2007) se interesaron por investigar el bienestar subjetivo y la autoeficacia en dominios como la salud, relaciones afectivas y trabajo en 795 estudiantes, profesores y empleados de una universidad de Bogotá (Colombia). También se ha investigado la relación entre el nivel de bienestar subjetivo y los estilos de afrontamiento al estrés (Verdugo-Lucero et al., 2013).

La investigación realizada por Giménez, Vásquez y Hervás (2010) apunta a mirar la adolescencia no como una etapa problemática, sino como un recurso en proceso de desarrollo. Lo que indica que los investigadores se interesan por cómo potenciar, promover, estimular y conservar lo positivo del ser humano, buscando capacidades y potencialidades en sujetos sanos.

Los investigadores han hallado recursos o activos con los que cuentan las personas para sobreponerse o superar obstáculos. En esta línea —en Sevilla, España-, Oliva, Antolín, Estévez, y Pascual (2011) encontraron que, para jóvenes entre 12 y 17 años, la dimensión comunitaria es relevante no solo para la prevención de problemas, sino también para promover la satisfacción vital. Se evidencia que ya el interés no se centra solo en lo patológico o disfuncional del ser humano, sino que también le apuntan a encontrar cuáles son los recursos o elementos con los que cuentan las personas para sentir bienestar.

Es posible experimentar bienestar subjetivo a pesar de estar expuestos a eventos traumáticos, 
a sentimientos dolorosos, duelos, angustias, temores, terrores. La presente investigación se preguntó por cuáles son los recursos psicosociales asociados al bienestar subjetivo de adultos entre 25 y 35 años del Oriente Antioqueño, que experimentaron sentimiento de ausencia de alguno de los padres. Es una realidad que las nuevas dinámicas familiares, la manera como se constituyen las familias, el aumento del número de familias monoparentales y el ingreso de la mujer en el mercado laboral hacen que muchas personas crezcan en ausencia de uno o ambos padres o que, incluso en presencia de ambos, experimenten sentimientos de ausencia porque en algunos casos hay negligencia e indiferencia por parte de los padres.

Por lo anterior, en esta investigación se indagó sobre los recursos psicosociales asociados al bienestar subjetivo de personas, que experimentaron sentimientos de ausencia de alguno de los padres. Se considera que las personas que sienten bienestar subjetivo, a pesar del sentimiento de ausencia de alguno de los padres, se debe a que contaron con recursos psicosociales que les permitieron hacerle frente a esta ausencia y superar las dificultades familiares.

En cuanto al término psicosocial, ha sido utilizado por diferentes disciplinas, en diferentes contextos, en diversos modelos de intervención, por variadas investigaciones, por profesionales en su quehacer $y$, sin embargo, es un término del que no se ha construido una teoría, y no se le puede atribuir a ninguna disciplina ni modelo en particular. Lo psicosocial es una forma de comprender lo humano donde lo relacional y lo vincular, lo contextual y la interacción son relevantes, un enfoque en el que se elige mirar a las personas en relación con su entorno vital, con los otros, con la comunidad y, a su vez percibir a esta última como un todo que se relaciona con su entorno contextual, regional, con otras comunidades y con sus miembros individuales (Villa, 2012).

Carmona (2012) sintetiza lo psicosocial como lo que "reintroduce al ser humano, como persona, y agente activo, en sus roles y sus contextos vinculares, en el campo de las Ciencias Sociales" (p. 13). Fernández (2009), por su parte, entiende lo psicosocial como una situación en la que aparece la relacionalidad: lo que queda en medio de dos cosas, lo psico y lo social. Lo psicosocial permite mirar la realidad desde diferentes lupas, reconoce al sujeto en construcción y en relación, lo psicosocial es la posibilidad de descubrir las interacciones en el mundo social.

Para efectos de la presente investigación, se entendió por "recursos psicosociales" los elementos, factores de protección, capacidades, habilidades, instituciones, entidades, personas, elementos de los que disponen los sujetos en las diferentes relaciones, situaciones, vínculos, roles e interacciones que logran establecer, y que les permiten hacer frente y lograr bienestar subjetivo a pesar de haber experimentado sentimientos de ausencia de alguno de sus padres.

Los recursos con los que cuentan los sujetos pueden ser tanto internos como externos; una de las capacidades que ayuda a superar las adversidades y amortiguar los efectos de los golpes emocionales es la resiliencia. Amar y Berdugo de Gómez (2006) mencionan que figuras representativas como tíos, primos, docentes ayudan a disminuir los efectos por la ausencia o relaciones conflictivas con los padres. Así mismo, Amar, Kotliarenko y Abello (2003) encontraron que los niños víctimas de violencia intrafamiliar pueden ser resilientes y pueden tener un desarrollo psicosocial positivo.

\section{Método}

\section{Participantes}

La muestra estuvo integrada por nueve individuos de ambos sexos, quienes cumplían con los criterios de inclusión: adultos que experimentaron sentimientos de ausencia de alguno de los padres, de edades entre 25 y 35 años, que residían en el Oriente Antioqueño, manifestaron bienestar subjetivo y accedieron a participar de manera voluntaria.

La identificación de los sujetos se realizó mediante el muestreo no probabilístico de bola de nieve, en el cual un informante referenciaba 
a otro o indicaba quién podría conocer personas con las características requeridas. También por referencia de estudiantes y docentes de la Universidad Católica de Oriente.

Los sujetos que participaron en la investigación actualmente se encuentran laborando, cuatro de ellos terminaron la licenciatura en lenguas extranjeras y son docentes de inglés; uno es licenciado en educación física, trabaja como coordinador pedagógico, se encuentra casado con un hijo; dos, graduados de psicología, una de ellas con maestría, se encuentran casados y laboran en el área de la psicología, y otro más, cursando pregrado en psicología, que realiza las prácticas y labora como auxiliar en salud pública. Manifiestan que disfrutan de compartir tiempo con la familia y los amigos, hacer ejercicio, leer, entre otras actividades lúdicas y recreativas.

\section{Instrumentos}

Se utilizó la entrevista semiestructurada, en la cual se indagó por los sentimientos de ausencia, bienestar subjetivo y recursos psicosociales. También se aplicaron láminas del TAT (Test de Apercepción Temática) que indagaban por los sentimientos de ausencia: 1, 6BM, 7BM, 7GF, $6 \mathrm{GF}, 13 \mathrm{~V}$ y la 16 , según corresponda por el sexo. Este no se utilizó como prueba de personalidad en sí, sino como movilizador de la palabra a través de la proyección que se suscita.

El TAT es una herramienta que puede ser utilizada en el contexto investigativo para mirar rasgos de personalidad, puede aportar a los recursos psíquicos y permite mirar en los participantes de la investigación, como lo menciona la prueba, una radiografía sobre la percepción interna y externa que ellos tienen de la vida. Para la aplicación se seleccionaron 8 láminas (1, 6BM, 7BM, 6 GF, 7GF, 13B, 13G, 16). Estas permiten indagar de manera general en la personalidad de los sujetos, sus relaciones con las figuras paterna y materna y sus ausencias físicas o emocionales, relaciones familiares y entre hermanos, la percepción que tiene el sujeto de su mundo, de sus miedos infantiles o primitivos y su proyección hacia el futuro.

\section{Procedimiento}

Inicialmente se realizó entrevista a 12 adultos, quienes previamente habían manifestado cumplir con los requisitos para participar en la investigación; se les explicó el proyecto de investigación y se les preguntó si cumplían los criterios de inclusión; se contactó a nueve de ellos, a quienes se confirmó por medio de autorreportes, (es decir, se le pide información a personas acerca de ellas mismas) que cumplían con lo solicitado. Una vez acordado un primer encuentro, se diligenció el consentimiento informado y se concertó cita para la entrevista y aplicación del TAT.

Posterior a esto, se realizaron las entrevistas y el TAT con cada uno, con una duración de dos horas. Luego, se realizó la transcripción de las grabaciones en Word. Después se codificó la información en el paquete Atlas $\mathrm{Ti}$, donde se establecieron familias con cada una de las categorías, se realizaron citas y memos. También se elaboró tabla en Excel, la cual permitió comprensión y claridad frente a la información obtenida en ambos instrumentos.

\section{Consideraciones éticas}

Cuando los participantes firmaron el consentimiento informado, se les dio a conocer las consideraciones éticas, tales como confidencialidad, derecho al anonimato, voluntariedad, devolución de resultados y derecho a retirarse del estudio.

La investigación que se realizó se podrá replicar y permitirá construir proyectos ajustados y mejorar procedimientos que contribuyan a pensar las dinámicas familiares actuales, la calidad de vida, el bienestar subjetivo, bienestar psicológico deseable para promover condiciones para los hijos y padres con una relación costobeneficio favorable para los individuos y las instituciones sociales. 


\section{Resultados}

Los resultados emergieron de las entrevistas y las láminas del TAT, aplicadas a los nueve informantes seleccionados. Inicialmente surgieron más de 150 códigos a partir de los primeros análisis de las entrevistas y los TAT; luego fueron renombrados y agrupados con otros códigos, según las tres categorías establecidas: recursos psicosociales, bienestar subjetivo y sentimiento de ausencia. En la información recogida, la categoría de recursos psicosociales fue la que arrojó mayor número de códigos, en donde prevalecieron los recursos internos, la capacidad para alcanzar metas personales y la capacidad para asumir responsabilidades. Respecto al bienestar subjetivo, los sujetos hacen una evaluación de sus propias vidas, expresan que sienten dicho bienestar gracias a que han logrado cumplir sus sueños, han logrado independencia y autonomía, lo que les permite hablar sobre el proyecto de vida que han construido y los llena de satisfacción. También manifiestan que durante una gran parte de su vida sintieron la ausencia de alguno de sus padres, lo que en algún momento les generó dolor, soledad y angustia.

\section{Sentimientos de ausencia}

Es evidente en toda la investigación, desde los relatos de los informantes, que sí han vivido y experimentado la ausencia, unos por la madre, otros por el padre y otros por ambos. El sentimiento de ausencia es evidente, pues relatan el dolor, rabia, angustia, soledad, frustración que en algún momento experimentaron. "Pues lo que siento por mi papá..., pero sí, sí he sentido rabia con él en muchas oportunidades, he sentido mucha ausencia..." (Sujeto 6).

"...yo ni me acuerdo..., creo que llorar y ya, porque no había de otra, y afortunadamente teníamos mi otro papá, que él siempre nos apoyaba; pero sí, eso fue muy complicado, porque uno saber que estaba solo en el mundo..." (Sujeto 5).

Dos de las personas manifestaron haber sido víctimas de rechazo, de abuso, de ausencia, de negligencia por parte del padre y no por esto prolongaron el modelo o los patrones de violencia:

...una vez me puse a hablar con mi hermano y le conté un episodio muy maluco que a mí me paso con Horacio y fue que, una vez borracho, pues, intentó abusar de mí, me ahorcó; yo no le conté directamente a mi hermano, y yo: ah parce, a mí me pasó algo con Horacio y creo que a Henry también y me dijo algo como de que, y yo relacionando pues con (baja el tono de la voz) pues con los abusos sexuales. Y me dijo: ah parce, ni me pregunte, porque a mí también me pasó(Sujeto 9).

Este mismo sujeto manifiesta que no recurre a la violencia sino que prefiere diálogo y contar con personas cercanas que lo apoyen:

...pues yo no sé, pues uno mm, confianza; entonces es una persona con la que pueda hablar, contarle las cosas y apoyo así sea con palabras, que lo escuche a uno, alguien con quien uno se pueda desahogar tranquilamente y eso fue lo que yo encontré.

Cuatro informantes reconocen relaciones distantes con la madre y la preferencia de esta por los hermanos:

...hay cosas que a uno lo marcan y yo no soy rencoroso con mi mamá; pero mi mamá hizo un gesto bastante... que a mi toda la vida me va a marcar, y es: "yo no los voy a dejar a ellos por usted". En ese tonito de USTED (Sujeto 5).

También manifiestan sentir ausencia de la madre por relaciones distantes, y donde los abuelos $\mathrm{u}$ otras personas asumieron la crianza: "mi mamá está viva, manejamos una relación, pero muy distante; igual para mí ha sido muy difícil como conectar esos lazos, pues porque igual han sido muchos años en los que he estado separado de ella" (Sujeto 3).

Cinco informantes manifiestan que, a pesar de que conocieron a los padres y convivieron con ellos, los sentían irresponsables y negligentes en la crianza, hasta el punto en el que sentían que no contaban con una familia: "pero no, es absolutamente solo, si yo hoy no tengo con que comer, pues de pronto aparecerá un amigo o algo, 
pero yo con la familia, totalmente solo" (Sujeto 5).

Tres sujetos manifestaron que fueron fruto de embarazo no deseado o no planeados por los padres: "... de hecho, mi mamá me dice que ella había pensado en no tenerme" (Sujeto 8); "...en cuanto mi mamá quedó en embarazo, ella no quería tenerme, cierto; entonces ella hizo muchas cosas por tratar de evitar el embarazo, interrumpirlo, ella no quería tenerme" (Sujeto 3).

Dentro de las razones para la ausencia por muerte del padre aparecen tres informantes: "a él lo mataron, era conductor y un taxista le pegó un tiro por algo que pasó con los carros..." (Sujeto 7). Una informante nunca conoció al padre ni a la familia paterna: “...bueno, mi madre es madre soltera, yo nunca viví con mi papá, de hecho nunca lo conocí, no sé ni siquiera cuál es la familia de él..." (Sujeto 8).

Todos los participantes también anhelaban estar incluidos y reconocidos dentro de una familia: "yo me di cuenta que, hiciera lo que hiciera, ellos no cambiarían conmigo y me relajé" (Sujeto 1).

\section{Bienestar subjetivo}

Los sujetos expresan el bienestar subjetivo al hacer la evaluación de su vida, y se sienten satisfechos con los logros alcanzados o por haber sido capaces de superar adversidades y hacer elecciones socialmente aceptables y constructivas:

...me siento muy bien y muy tranquila con la vida que llevo; entonces, tengo un buen trabajo, estable, me encanta vivir sola... tengo la universidad, me encanta esta universidad, me encanta la carrera que elegí, estoy enamorada y me voy a casar (Sujeto 7).

"...me siento bien en muchos aspectos: académicamente me siento bien, con mi esposo, con mis amigos y en lo personal, rodeada de personas muy humildes y sencillas. Rodeada de todo ese afecto me siento muy bien" (Sujeto 6).

Logran estudiar, formar una familia, conseguir amigos, mantener trabajos estables y perdonar a aquellas personas que consideraron negligentes, irresponsables y que incluso se mostraron indiferentes cuando los necesitaron, se proyectan a mediano y largo plazo:

\begin{abstract}
...terminar la licenciatura, yo ya me puse a buscar terminar la licenciatura y no, apenas la termine me tengo que hacer un certificado en la Eafit, que es de metodología experiencial en inglés. Usted toma CELTA, presenta después que lo certifiquen obviamente, pasarlo, presenta como uno o dos años de experiencia; certifica el grado que se llama CELTA. CELTA aplica a nivel mundial, entonces, es casi imposible que a usted no le den trabajo en cualquier parte del mundo. Entonces es como eso; obviamente me gustaría hacer una maestría en metodologías y en algún momento me gustaría sentarme a escribir, uno sobre aprendizaje de lenguas y dos, cualquier barrabasada (Sujeto 9).
\end{abstract}

También manifiestan estar en construcción de la felicidad: "ser feliz no es estar en un bar, comprar un viaje, ser feliz, es más como compartirles a las personas internas que uno tiene, estar tranquilo; para mí eso es estar feliz, tener mucha tranquilidad, mucha paz interior" (Sujeto 8).

\section{Recursos psicosociales}

En estos sujetos se comprueba que contaron con recursos psicosociales que les permitieron hacerle frente a la ausencia de los padres y construir bienestar. Se identificó que los sujetos contaron con personas representativas; unos con la mamá, cuando el papá era negligente o estaba ausente, donde ella era quien motivaba y brindaba apoyo y estabilidad: "pues mi mamá ha ejercido un rol fuerte de crianza, ha sido en esos periodos críticos, fue siempre como ahí, y eso me ayudó a estar bien formado y a uno razonar sobre muchas cosas, que deben ser importantes para el futuro de uno" (Sujeto 4). La pareja también es reconocida como quien brindaba sostén: "el acompañamiento de mi esposo; yo estoy con él desde los 15 años, y yo a él se lo reconozco, de no haber tenido una relación seria con él, me hubiera perdido" (Sujeto 1). También contaron 
con figuras significativas que los acompañaron y contribuyeron a la construcción de proyecto de vida familiar y personal, como en el caso del Sujeto 1.

En dos sujetos, el padrastro fue relevante porque brindó compañía y apoyo económico: "afortunadamente, pues Dios es muy grande y a mí me dio otro papá, o sea, no el biológico... fue el papá de mi hermana que también me quiso muchísimo" (Sujeto 5). En otros informantes hubo tíos o docentes que incentivaron la formación académica y la inserción laboral: "salió la licenciatura y fue muy chévere porque los mismos docentes del diplomado le decían a uno susurrando: 'métase a la licenciatura, métase, necesitamos personas como usted' " (Sujeto 9).

También se encontró que las instituciones, como los grupos deportivos, recreativos, culturales, grupos de apoyo social como bomberos o defensa civil, se convirtieron en la posibilidad de canalizar los sentimientos y ofrecer opciones para enfrentar la soledad que pudieran experimentar: “...yo hoy sigo siendo parte de la Defensa Civil, llevo 19 años, he tenido muchas dificultades por cambios, pero yo siento que esos grupos a uno definitivamente si uno lo sabe aprovechar lo forman a uno y lo apoyan demasiado" (Sujeto 3).

...pertenecí al grupo de baloncesto, pertenecí a un club de microfútbol, ya después de eso pues me dedicaba a jugar microfútbol solamente y así en recochita por ejemplo, ya los fines de semana sí salía a montar bicicleta o también los fines de semana me iba para donde, para donde una tía mía que vive pues en una finca por acá cerca, acá en Rionegro; y, y ya pues jugaba con un primo mío o salíamos a Comfama. También los fines de semana montábamos bicicleta; muchas cosas también así como a nivel deportivo recreativo. (Sujeto 4)

Hay unos recursos psicológicos con los cuales los sujetos contaron, como la capacidad de resiliencia, lo que les permitió superar las adversidades tanto económicas como emocionales: "...hay muchas situaciones en las que implica tristeza, como la partida de un ser querido, pero eeeh, no sé, es algo muy momentáneo y que uno enfrenta; uno sabe que, bueno, pasó esto pero la vida continúa, mi vida continúa, es afrontar la situación, la dificultad y seguir" (Sujeto 2). La autonomía y capacidad para alcanzar metas personales que favorecía ser selectivos y buscar y elegir las oportunidades que los llevaran a lograr los objetivos: "iyo me di todo!, o sea, yo luché por lo que tengo, busqué todo e hice todo, estoy estudiando y trabajando" (Sujeto 9).

En la mayoría de ellos era evidente su capacidad para hacer revisión y lectura de contexto y situación, pues fueron capaces de planificar y orientar sus esfuerzos hacia sus deseos y alcanzar metas personales: "a pesar de que vivía en un barrio muy muy malo..., yo quería estudiar y yo quería ser una profesional” (Sujeto 5). “... me tocó costearme el bachillerato, pues no había plata, situaciones complicadas como definir una situación militar; yo logro graduarme primero que mis hermanos de bachillerato" (Sujeto 3).

En los resultados se comprobó que todos los sujetos experimentaron ausencia de alguno o de ambos padres; sin embargo, también supieron aprovechar los recursos con los que contaban, elaborar la pérdida y apuntar a opciones y proyectos de vida que les generaron gratificación, satisfacción y bienestar subjetivo.

\section{Discusión}

Los participantes en la investigación expresan que experimentaron sentimientos de ausencia de alguno o de ambos padres; sin embargo, contrario a lo que plantean Vallejo, Sánchez y Sánchez (2004), quienes le dan toda la relevancia a las relaciones entre padres e hijos como determinantes para establecer relaciones durante toda la vida, los sujetos de este estudio lograron construir bienestar subjetivo y establecer relaciones duraderas y estables con otras personas.

Como lo evidencian los resultados, se corrobora lo encontrado por Muñoz, Gómez y Santamaría-Ogliastri (2008) respecto a los sentimientos y pensamientos que se presentaron después de la separación de los padres, entre estos: inseguridad, preocupación por el futuro, 
temores, ansiedad, sentimiento de rabia, tristeza y resentimiento. Contrario a lo reportado por Botero (2008), en el presente estudio se encontró que, a pesar de que varios de los sujetos fueron víctimas de rechazo, de abuso, de ausencia, de negligencia por parte del padre, no por esto prolongaron el modelo o los patrones de violencia. En esta misma línea, a diferencia de lo planteado por Gracia, Lila y Musitu (2005), los sujetos de la presente investigación encontraron aceptación y un ambiente benévolo en otros espacios diferentes a los familiares, y no tienden a percibir el mundo como amenazador y hostil a pesar de que fueron rechazados. Ante la adversidad, una persona puede tener o no recursos psicosociales necesarios para desarrollarse y llevar adelante una vida (García, 2016).

Por otro lado, acorde a lo planteado por Diener (2002, citado por Gómez et al., 2007) y por Muñoz (2007), el bienestar subjetivo en los sujetos de estudio se evidencia en las elecciones que hicieron, pues lograron establecer relaciones estables y duraderas, conformar y consolidar una relación de pareja, acceder a la formación académica, ingresar al mundo laboral y conservar la estabilidad; también lograron mayores ingresos económicos, lo que les ha permitido mejorar la calidad de vida. Estas personas hacen evaluaciones positivas de sus vidas, de los logros y metas alcanzadas, se consideran con capacidades y aptitudes que les ha permitido avanzar y construir proyectos de vida satisfactorios, se consideran eficaces en lo que hacen y son positivos y optimistas frente a sus planes.

De acuerdo con lo planteado por Cáceres (2006), Escarti, Buelga, Gutiérrez y Pascual (2009), Oliva et al. (2010) y Rosenfeld (2009), estos sujetos contaron con recursos personales, familiares, escolares o comunitarios que supieron aprovechar para construir proyectos de vida; instituciones como la escuela, grupos deportivos, recreativos, culturales u otros familiares. Dentro de los recursos externos, y como lo plantean López y Loaiza (2009), también contaron con personas representativas como la madre cuando hubo ausencia del padre-, tías, tíos, abuelos, docentes, amigos, padrastros y la pareja, quienes los acompañaron y brindaron apoyo. Es posible, entonces, afirmar con Lacunza (2009), que las habilidades sociales se constituyeron en un recurso que favorece los comportamientos sociales, el bienestar, la adaptación y la aceptación de los otros. Estas personas se mostraron como resilientes, lo que según García (2016), puede constituir un conjunto de habilidades y competencias que los capacitan para enfrentar positivamente experiencias negativas, y que a pesar de condiciones vitales difíciles, las personas pueden crecer y autorrealizarse.

El acceso a educación superior se convierte en un factor clave que potencia el desarrollo y posibilita la construcción de bienestar, pues disminuye los factores de riesgo a los cuales están expuestos todos los participantes de la investigación, lo que corrobora lo planteado por Navarro (2009, citado por De la Cruz, Feu, \& Vizuete, 2013) quienes plantean que la educación contribuye a la generación de bienestar en la población y permite el empoderamiento y la obtención de recursos para resolver los problemas.

De la investigación se puede concluir que fueron los recursos psicosociales los que mediaron para que los jóvenes, a pesar de haber experimentado ausencia de alguno de los padres, construyeran bienestar. Los hechos confirman que las personas tienden a buscar otros caminos, alternativas, salidas, respuestas que permitan contrarrestar estos sentimientos.

Efectivamente, estos informantes tuvieron padres o madres en algunos casos negligentes en la crianza, en otros irresponsables con las obligaciones económicas, padres maltratadores, abusadores y que rechazaban a sus hijos; por lo que encontraron en otras personas o lugares el afecto y estabilidad que los padres no les suministraron. Contaron con figuras significativas que los acompañaron y contribuyeron a la construcción de proyecto de vida; por lo que puede decirse que personas diferentes a los padres pueden ser relevantes para la satisfacción vital. 
Se resalta que contaron con instituciones $\mathrm{y}$ redes de apoyo que fueron fundamentales y sirvieron como canalizadores de angustias, rabias, dolor, tristeza, soledad, sentimientos que experimentaron por la ausencia de los padres. Estas entidades también contribuyeron para fortalecer y encauzar sus planes a futuro.

En sus relatos, los informantes muestran que contaron con recursos internos como la capacidad de resiliencia, la capacidad de proyectarse, de revisión y planificación y de autonomía, en los cuales se evidencia que, con frecuencia, hablan de sus metas, logros y su interés por la formación profesional. Estos elementos les han permitido superar cada situación adversa que se presentaba con su familia. En sus realidades se muestra que algunos culminaron sus estudios y están ejerciendo, y otros están en proceso y con el deseo de terminar sus carreras.

\section{Agradecimientos}

Los autores agradecen a los nueve participantes, por su disponibilidad y disposición para suministrar información para el proyecto de investigación, "Recursos Psicosociales asociados al Bienestar Subjetivo de adultos entre 25 y 35 años del oriente antioqueño, que experimentaron sentimientos de ausencia de alguno de sus padres", el cual se llevó a cabo entre mayo de 2013 a mayo de 2015.

\section{Referencias}

Amar, J. J., \& Berdugo de Gómez, M. (2006). Vínculos de apego en niños víctimas de la violencia intrafamiliar. Psicología desde el caribe, 18, 108-122.

Amar, J. J, Kotliarenko, M., \& Abello, R. (2003). Factores psicosociales asociados con la resiliencia en niños colombianos víctimas de violencia intrafamiliar. Investigación y Desarrollo, 18 (1), 162-197.

Botero, H. (2008). Cuando papá no está: la ausencia del padre como un factor generador de violencia. Revista de la
Asociación Psicoanalítica Colombiana, 20(1), 60-91.

Carmona, J. (2012, abril) ¿Qué es lo psicosocial? Cinco principios de respuesta a la pregunta. Ponencia presentada en el seminario "iQué es lo Psicosocial?". Medellín: Fundación Universitaria Luis Amigó.

Cáceres, N. E. (2006). Experiencia perceptiva de sí mismo, de su familia y comunidad en un grupo de jóvenes del barrio El Vergel de la Comuna 13, de Santiago de Cali. Pensamiento Psicológico, 2(7), 149-168.

Cebotarev, E. (2003). Familia, socialización y nueva paternidad. Revista Latinoamericana de Ciencias Sociales, Niñez y Juventud, 1(2), 2-18. Recuperado de http://biblioteca.clacs o.edu.ar/Colombia/alianza-cinde-umz/201 31004011637/art.NoraC.pdf

De la Cruz-Sánchez, E., Feu, S., \& VizueteCarrizosa, M. (2013). El nivel educativo como factor asociado al bienestar subjetivo y la salud mental en la población española. Universitas Psychologica, 12(1), 31-40.

Escarti, A., Buelga, S., Gutiérrez, M., \& Pascual, C. (2009). El desarrollo positivo a través de la actividad física y el deporte: Programa de responsabilidad personal y social. Revista de Psicología General y Aplicada, 12, 45-52.

Fernández, P. (2009). Lo psicosocial. Revista desdisciplinada de Psicología Social, 1, 42-47.

García, J. (2016). Afrontando la adversidad. Resiliencia, optimismo y sentido de la vida. Sociedad Latina de Comunicación Social - edición no verbal. Recuperado de https://www.researchgate.net/publicatio $\mathrm{n} / 311861820$

Giménez, M., Vázquez, C., \& Hervás. G. (2010). El análisis de las fortalezas psicológicas en la adolescencia: más allá de los modelos de vulnerabilidad. Psychology, Society $\mathfrak{B}$ Education, 2(2), 97-116.

Gómez, V., Villegas, C., Barrera, F., \& Cruz, J. E. (2007). Factores predictores de bienestar subjetivo en una muestra colombiana. Revista Latinoamericana de Psicología, 39(2), 312-325.

Gracia, E., Lila, M., \& Musitu, G. (2005). Rechazo parental y ajuste psicológico y 
social de los hijos. Salud Mental, 28(2), 73-81.

Lacunza, A. B. (2009). Las habilidades sociales como recursos para el desarrollo de fortalezas en la infancia. Psicodebate Psicología, Cultura y Sociedad, 10, 231-248. Recuperado de http://dspace.palermo.edu/dspace/bitstr eam/10226/586/1/10Psico_13.pdf

López, L. M., \& Loaiza, M. O. (2009). Padres y madres migrantes internaciones y su familia: oportunidades y nuevos desafíos. Revista Latinoamericana de Ciencias Sociales, Niñez y Juventud, 7(2), 873-860.

Muñoz, C. (2007). Perspectiva Psicológica de Bienestar Subjetivo. Psicogente, 18, 163-173.

Muñoz, M., Gómez, P., \& Santamaría-Ogliastri, C. (2008). Pensamientos y sentimientos reportados por los niños ante la separación de sus padres. Universitas Psychologica, 7(2), 347-356.

Oliva, A., Ríos, M., Antolín, L., Parra, A., Hernando, A., \& Pertegal, M. A. (2010). Más allá del déficit: construyendo un modelo de desarrollo positivo adolescente. Sevilla: Fundación Infancia y Aprendizaje.

Oliva, A., Antolin, L., Estévez, R., \& Pascual, D. M. (2011). Activos del barrio y ajuste adolescente. Sevilla: Universidad de Sevilla.

Pérez, C. H., Bonnefoy, C., Cabrera, A., Peine, S., Muños, C., Baquedano, M., \& Jiménez, J. (2011). Análisis, desde la psicología positiva, de la salud mental en estudiantes universitarios de primer año de Concepción - Chile. Avances en Psicología Latinoamericana, 29(1), 148-160.

Profamilia. (2010). Encuesta de Demografía y Salud - Ends. Recuperado de http://www.profamilia.org.co/encuestas/ Profamilia/Profamilia/index.php?option $=\mathrm{c}$ om_content\&view $=$ article\&id $=62 \&$ Item $\mathrm{id}=9$

Profamilia. (2015). Encuesta de Demografía y Salud - Ends. Recuperado de https://profam ilia.org.co//investigaciones/ends/

Rosenfeld, E. (2009). Los procesos de producción artística como espacios para el desarrollo positivo de los jóvenes. Revista Interuniversitaria de Formación de Profesorado, 23(3), 181-202.

Semana. (2016, 11 de mayo). Los papás importan, pero no tanto. Recuperado de http://www.semana.com/vida-moderna/ articulo/crianza-de-los-ninos-influencia-de -los-padres-en-el-desarrollo/504438

The Family Watch. (2015). Informe TFW 2105-1 La importancia de la figura paterna en la educación de los hijos: estabilidad familiar y desarrollo social. Recuperado de http://www.thefamilywatch.org/wp-cont ent/uploads/Informe20151.pdf

Vallejo, R., Sánchez, F., \& Sánchez, P. (2004). Separación o divorcio: Trastornos psicológicos en los padres y los hijos. Revista de la Asociación Española de Neuropsiquiatría, 92, 91-110.

Verdugo-Lucero, J. L., Ponce de León-Pegaza, B. G., Guardado-Llamas, R.E, Meda-Lara, R. M., Uribe-Albarado, J. I., \& GuzmánNuñiz, J. (2013). Estilos de afrontamiento al estrés y bienestar subjetivo en adolescentes y jóvenes. Revista Latinoamericana de Ciencias Sociales, Niñez y Juventud, 11(1), 79-91.

Villa, J. D. (2012). La acción y el enfoque psicosocial de la intervención en contextos sociales: ipodemos pasar de la moda a la precisión teórica, epistemológica y metodológica? El Ágora, 12 (2), 349-365.

\section{Notas}

* Artículo de investigación. 